\title{
Glial Fibrillary Tangles and JAK/STAT-Mediated Glial and Neuronal Cell Death in a Drosophila Model of Glial Tauopathy
}

\author{
Kenneth J. Colodner and Mel B. Feany \\ Program in Neuroscience, Harvard Medical School, Department of Pathology, Brigham and Women's Hospital, Boston, Massachusetts 02115
}

\begin{abstract}
A subset of neurodegenerative tauopathies is characterized by abundant filamentous inclusions of hyperphosphorylated tau in both neurons and glia. Although the contribution of neuronal tau to behavioral changes and neuronal loss in neurodegenerative diseases has been studied extensively, the functional consequences of tau deposition in glial cells have been less well characterized. To investigate the role of abnormal tau accumulation and aggregation in glial cells, we created a Drosophila model of glial tauopathy by expressing human wild-type tau in adult fly glial cells. Glial expression of tau resulted in robust aggregation of phosphorylated tau into fibrillary inclusions similar to human glial tangles. Tangle formation was accompanied by shortened lifespan and age-dependent apoptotic cell death of both glia and neurons. Genetic manipulation of Janus kinase/signal transducer and activator of transcription (JAK/STAT) signaling modified toxicity of glial tau. We also identified a synergistic interaction of combined tau expression in neurons and glial cells. In summary, we present a genetically tractable model of glial fibrillary tau tangle formation and identify JAK/STAT signaling as mediating the death of both glia and neurons in this model.
\end{abstract}

\section{Introduction}

Tauopathies, including Alzheimer's disease, are characterized by filamentous inclusions composed of hyperphosphorylated and abnormally aggregated tau, a microtubule-associated protein (Buée et al., 2000). Mutations in the tau gene cause the autosomal dominant tauopathy frontotemporal dementia with parkinsonism linked to chromosome 17 (FTDP-17) (Hutton et al., 1998; Spillantini et al., 1998; D'Souza et al., 1999; Mirra et al., 1999; Tolnay et al., 2000). Development of other tauopathies, including corticobasal degeneration and progressive supranuclear palsy, is influenced by specific tau haplotypes (Conrad et al., 1997; Houlden et al., 2001; Goedert and Jakes, 2005). Postmortem analysis of brains from patients with progressive supranuclear palsy, corticobasal degeneration, and related tauopathies has demonstrated that numerous fibrillar tau inclusions are present in both neurons and glia (Chin and Goldman, 1996; Ballatore et al., 2007). Glial tau inclusions can be highly characteristic of particular tauopathies (Feany and Dickson, 1995; Ikeda et al., 1995; Berry et al., 2001). In Alzheimer's disease, glial tau pathology can also be found in astrocytes and oligodendrocytes (Papasozomenos, 1989a,b; Nakano et al., 1992; Nishimura et al., 1995), although less prominently than neuronal tau deposition.

A number of groups have demonstrated that the overexpression of wild-type and FTDP-17-associated mutant tau in neurons produces neurotoxicity in murine (Ishihara et al., 1999; Spittaels

\footnotetext{
Received May 15, 2010; revised Sept. 15, 2010; accepted Sept. 17, 2010. We thank members of the Feany laboratory for valuable discussions.

Correspondence should be addressed to Mel B. Feany, Department of Pathology, Brigham and Women's Hospital,

77 Louis Pasteur Avenue, Room 630, Boston, MA 02115. E-mail: mel_feany@hms.harvard.edu.

DOI:10.1523/JNEUROSCI.2491-10.2010

Copyright $\odot 2010$ the authors $\quad 0270-6474 / 10 / 3016102-12 \$ 15.00 / 0$
}

et al., 1999; Lewis et al., 2000; Probst et al., 2000), Drosophila (Wittmann et al., 2001; Jackson et al., 2002), and Caenorhabditis elegans (Kraemer et al., 2003; Miyasaka et al., 2005; Brandt et al., 2009) tauopathy models. Moreover, transgenic mice overexpressing wild-type tau in astrocytes (Forman et al., 2005) and FTDP-17-associated mutant tau in oligodendrocytes (Higuchi et al., 2005) revealed that expression of human tau in murine glial cells disrupts normal glial function (Dabir et al., 2006) and produces neurodegenerative changes.

The utility of using Drosophila to model human neurodegenerative disorders lies in the ability to genetically dissect mechanisms of toxicity (Muqit and Feany, 2002; Bonini and Fortini, 2003; Marsh and Thompson, 2006). Previous studies in the fly have identified key mediators of tau toxicity in neurons (Jackson et al., 2002; Nishimura et al., 2004; Khurana et al., 2006; DiasSantagata et al., 2007; Fulga et al., 2007), as well as important pathogenic mechanisms in diseases ranging from Parkinson's disease (Auluck et al., 2002; Chen and Feany, 2005; Periquet et al., 2007) to polyglutamine disorders (Warrick et al., 1999; Steffan et al., 2001). Because Drosophila glial cells perform similar functions to their vertebrate counterparts (Freeman and Doherty, 2006) and are critical for maintaining neuronal viability (Xiong et al., 1994; Rival et al., 2004; Lievens et al., 2005), we sought to develop a model of glial tauopathy in Drosophila.

To determine whether expression of human wild-type tau in Drosophila glial cells is toxic, we expressed tau in adult fly glia. We find that glial-specific expression of human tau decreases lifespan and results in age-dependent glial fibrillary tangle formation. We observe both cell-autonomous glial and non-cell-autonomous neuronal cell death that is mediated though the Janus kinase/ signal transducer and activator of transcription (JAK/STAT) sig- 
naling cascade. Finally, we show a synergistic enhancement of toxicity with expression of wild-type human tau in both neurons and glia.

\section{Materials and Methods}

Drosophila stocks and genetics. Human wild-type tau containing no $\mathrm{N}$-terminal inserts and four microtubule binding domains $(0 \mathrm{~N}, 4 \mathrm{R})$ was expressed in adult glia by recombining the glial-specific driver repoGAL4 with a ubiquitously expressed temperature-sensitive allele of GAL80 (tub-GAL80 ${ }^{T S}$ ) (McGuire et al., 2003). To minimize transgene expression and prevent lethality produced by expression of tau during development, all flies were crossed at $17^{\circ} \mathrm{C}$. During eclosion, flies were aged at $30^{\circ} \mathrm{C}$ in the experiments described in Figures 1-4 and 6-7 and supplemental Figures S3-S6 (available at www.jneurosci.org as supplemental material). In other experiments, the temperature at which the flies were aged was varied to modulate transgenic expression of tau as indicated in the figure legends. In Figures 1-5 and supplemental Figures S1-S4 (available at www.jneurosci.org as supplemental material), the genotype of the experimental (glial tau transgenic) flies was $U A S-t a u^{W T}$ / repo-GAL4 tub-GAL80 ${ }^{T S}$, whereas control flies were of the genotype repo-GAL4 tub-GAL80TS/+. In other figures, the genotypes varied and are detailed in the figure legends. Control flies in all experiments were as closely related to the experimental tau transgenic flies as possible, typically only lacking the UAS-tau ${ }^{W T}$ transgene. Detailed genotypes are provided in each figure legend.

The following fly stocks were used: repo-GAL4 (Sepp and Auld, 1999); UAS-Socs36E (Callus and Mathey-Prevot, 2002); 10XSTAT-GFP (Bach et al., 2007); UAS-STAT-GFP (Brown et al., 2006); UAS-Socs36E-IR (Vienna Drosophila RNAi Center, Vienna, Austria); and elav-GAL4, $h o p^{2}$, tub-GAL80 ${ }^{T S}$ (Bloomington Stock Center, Indiana University, Bloomington, IN).

Lifespan analysis. Crosses were performed at $17^{\circ} \mathrm{C}$, and newly eclosed flies were collected and aged at either $25^{\circ} \mathrm{C}$ or $30^{\circ} \mathrm{C}$. Three hundred flies were aged in individual vials containing no more than 30 flies each and assayed for longevity (Wittmann et al., 2001). Food was changed every $1-2 \mathrm{~d}$, and the number of dead flies was recorded.

Immunohistochemistry, immunofluorescence, and terminal deoxynucleotidyl transferase-mediated biotinylated UTP nick end labeling analysis. For paraffin histology and immunostaining, adult flies were fixed in formalin and embedded in paraffin. Serial frontal sections $(4 \mu \mathrm{m})$ were prepared through the entire fly brain. Slides were processed through xylene, ethanol, and into water. Antigen retrieval by boiling in sodium citrate, $\mathrm{pH}$ 6.0, was used before immunostaining on paraffin sections. In some studies, fresh brains were fixed directly in formalin or methanol. Slides were blocked in PBS containing 0.3\% Triton X-100 and 5\% milk for $1 \mathrm{~h}$. Immunostaining was performed using the following primary antibodies at the specified dilutions: mouse anti-reversed polarity (repo) (1:25; Developmental Studies Hybridoma Bank, Iowa City, IA), rat antirepo (1:25; (Campbell et al., 1994), anti-choline acetyltransferase (ChAT) (1:250) (Yasuyama et al., 1995), anti-activated caspase-3 (1:50; Trevigen), mouse anti-green fluorescent protein (GFP) (1:50; Clontech), rabbit anti-GFP (1:50; Invitrogen), AT8 (1:1000; Thermo Scientific Pierce), AT100 (1:1000; Thermo Scientific Pierce), anti-PThr212 (1:500; Invitrogen), anti-PSer214 (1:500; Invitrogen), PHF1 (1:100; P. Davies, Albert Einstein College of Medicine, Bronx, NY), MC1 (1:100; P. Davies), and Alz50 (1:50; P. Davies). For immunofluorescence, the appropriate fluorescent secondary antibodies (Alexa Fluor 488, Alexa Fluor 555; Invitrogen) were diluted at 1:200. For immunohistochemistry, biotinconjugated secondary antibodies (1:200; Southern Biotechnology) and avidin-biotin-peroxidase complex (Vectastain Elite; Vector Laboratories) staining was performed. Histochemical detection was performed by developing with DAB (Sigma-Aldrich).

Apoptotic cell death was visualized using terminal deoxynucleotidyl transferase-mediated biotinylated UTP nick end labeling (TUNEL) according to the instructions of the manufacturer (TdT FragEL DNA fragmentation kit; Calbiochem), with an additional avidin-biotin-peroxidase amplification step. At least eight hemibrains in the region from the midline to the outer optic chiasm (Tix et al., 1997) were analyzed, and the number of
TUNEL-positive cells throughout the entire hemibrain was quantified. For double-labeled TUNEL studies, avidin-biotin-peroxidase complex and streptavidin-coupled Alexa Fluor 488 (1:200) was used to identify TUNELpositive cells, and subsequent immunofluorescence detection using antirepo or anti-choline acetyltransferase was used to identify the cells with TUNEL-positive nuclei.

Bielschowsky silver stain and thioflavin-S staining were performed using standard techniques (Yamamoto and Hirano, 1986).

Sarkosyl extraction. Sarkosyl extraction was performed as described (Goedert et al., 1992). Briefly, 50 heads from 1-, 10-, and 20-d-old flies expressing tau in glia and 20-d-old control flies were homogenized in 50 $\mu \mathrm{l}$ of homogenization buffer (in mM: $15 \mathrm{NaCl}, 25$ Tris- $\mathrm{HCl}$ at $\mathrm{pH} 7.4,1$ EGTA, and 1 EDTA) containing protease inhibitors (Roche Diagnostics). After a brief centrifugation to pellet cuticle and other large debris, the homogenate was spun for $1 \mathrm{~h}$ at $100,000 \times g$, and the supernatant was saved as soluble fraction. The pellet was resuspended in salt/sucrose buffer ( $10 \%$ sucrose, $0.8 \mathrm{M} \mathrm{NaCl}, 10 \mathrm{~mm}$ Tris- $\mathrm{HCl}$ at $\mathrm{pH} 7.4$, and $1 \mathrm{~mm}$ EGTA) containing a protease inhibitor cocktail and centrifuged for 30 $\min$ at $15,000 \times g$. Sarkosyl was added to the supernatant (to make $1 \%$ Sarkosyl) and incubated at $37^{\circ} \mathrm{C}$ for $1 \mathrm{~h}$ and then centrifuged for $2 \mathrm{~h}$ at $100,000 \times g$. The Sarkosyl-insoluble pellet was then resuspended in $15 \mu \mathrm{l}$ of homogenization buffer. This fraction was then subjected to both immunoelectron microscopy and Western blot analysis. Equivalent volumes of buffers were used on each experimental condition to ensure similar processing.

For immunostaining of Sarkosyl fractions, an aliquot of the Sarkosylinsoluble pellet fraction isolated from 20-d-old glial tau transgenic and control flies was absorbed onto Formvar-coated, carbon-stabilized, copper grids and air dried. Grids were blocked in PBS with 1\% BSA for 30 min and incubated with a phosphorylation-independent polyclonal antitau antibody (1:50; Dako) for $3 \mathrm{~h}$, washed with PBS, and incubated with $10 \mathrm{~nm}$ gold-conjugated secondary antibody (1:50; Sigma-Aldrich) for $2.5 \mathrm{~h}$. The grids were washed with $\mathrm{dH}_{2} \mathrm{O}$ and negatively stained with $2 \%$ uranyl acetate, washed, air dried, and examined on a Tecnai $\mathrm{G}^{2}$ Spirit BioTWIN transmission electron microscope.

Phosphatase treatment. Heads from 10- and 30-d-old glial tau transgenic flies were homogenized in $1 \times$ lambda phosphatase buffer $(\mathrm{New}$ England Biolabs) containing a protease inhibitor cocktail. Homogenates were incubated with or without lambda protein phosphatase for $3 \mathrm{~h}$ at $37^{\circ} \mathrm{C}$. Specimens were then subjected to Western blot analysis.

Western blots. Adult Drosophila heads were homogenized in, or Sarkosyl-extracted fractions were combined with, $2 \times$ Laemli's buffer (Sigma-Aldrich). Samples were boiled for $10 \mathrm{~min}$, briefly centrifuged, and subjected to SDS-PAGE in 10\% separating gels (Cambrex). Proteins were transferred to nitrocellulose membranes (Bio-Rad), blocked in $2 \%$ milk in PBS with $0.05 \%$ Tween 20, and immunoblotted using one of the following antibodies: a phosphorylation-independent rabbit polyclonal anti-tau antibody (1:107 ; Dako), AT8 (1:100,000; Thermo Scientific Pierce), AT100 (1:1000; Thermo Scientific Pierce), AT270 (1:100,000; Thermo Scientific Pierce), AT180 (1:10,000; Innogenetics), PHF1 (1: 1000; P. Davies), anti-actin (1:50,000; Sigma-Aldrich), anti-Nrv (1:100; Developmental Studies Hybridoma Bank), or anti-GFP (1:50,000; Clontech). The appropriate anti-mouse or anti-rabbit horseradish peroxidase-conjugated secondary antibody (1:50,000; Southern Biotechnology) was applied, and signal was detected by chemiluminescence (Alpha Innotech). Ponceau S staining was used to evaluate protein loading and transfer in all Western blots. Western blots were also reprobed with antibodies recognizing actin or the $\mathrm{Na}^{+}, \mathrm{K}^{+}$-ATPase Nrv, a widely expressed protein in the Drosophila brain (Sun et al., 1998), to illustrate equivalent protein loading.

Electron microscopy. Brains from 25-d-old glial tau transgenic and control flies were dissected out of the cuticle and fixed in $2.5 \%$ glutaraldehyde (Polysciences). Brains were then incubated in 1\% osmium tetroxide (Electron Microscopy Sciences) $/ 1.5 \%$ potassium ferrocyanide (MP Biomedicals) for $1 \mathrm{~h}, 1 \%$ uranyl acetate for $30 \mathrm{~min}$, and then processed through 70, 90, and 100\% ethanol solutions. Brains were then incubated in propyleneoxide for $1 \mathrm{~h}$ and embedded in Epon and allowed to polymerize for $2 \mathrm{~d}$ at $60^{\circ} \mathrm{C}$. Ninety-five nanometer sections were cut and 
examined with a Tecnai $\mathrm{G}^{2}$ Spirit BioTWIN transmission electron microscope at an accelerating voltage of $80 \mathrm{kV}$.

For immunostaining, brains of 25-d-old glial tau transgenic and control flies were dissected and fixed in $4 \%$ paraformaldehyde (Electron Microscopy Sciences) and $0.5 \%$ glutaraldehyde (Polysciences) for 10 $\mathrm{min}$. Brains were washed with PBS and incubated in $0.1 \mathrm{M}$ glycine and then processed through 30,50 , and $70 \%$ ethanol washes. Brains were then embedded in LR-white resin (Electron Microscopy Sciences) and were allowed to polymerize for $2 \mathrm{~d}$ at $59^{\circ} \mathrm{C}$. Ninety-five nanometer sections were stained with AT8 (1:10; Thermo Scientific Pierce) for $4 \mathrm{~h}$, washed with PBS, and incubated with $5 \mathrm{~nm}$ gold-conjugated secondary antibody (1:50; Sigma-Aldrich) for $2 \mathrm{~h}$. Sections were negatively stained with $2 \%$ uranyl acetate and Reynold's lead citrate and examined, as above. We used AT8 rather than AT100 for the immunoelectron microscopy because we found that AT8 was more readily detected than AT100 after glutaraldehyde fixation.

Quantification of STAT-GFP levels. Densitometric analysis of GFP levels was performed using FluoroChemHD2 software on Western blots probed with Clontech antibody recognizing GFP $(1: 50,000)$. The results represent the average of at least six animals per genotype, in which protein homogenates were prepared and assayed separately from at least six individual fly heads per genotype.

\section{Results}

\section{Expression of tau in Drosophila glial cells reduces lifespan}

To express human wild-type tau in Drosophila glial cells, we used the bipartite galactosidase-4 (GAL4)/upstream activating sequence (UAS) transcriptional-transactivation system (Brand and Perrimon, 1993) and the repo-GAL4 driver. repo-GAL4, a widely used Drosophila glial driver, is activated during embryogenesis and is expressed in most glial cells in the Drosophila CNS (Sepp et al., 2001; Freeman et al., 2003). When human tau was expressed with the repo-GAL4 driver pharate, adult lethality was observed. No adults eclosed, even when the cross was performed at $17^{\circ} \mathrm{C}$ to minimize transgene expression. To circumvent developmental toxicity of tau, we used a ubiquitously expressed, temperature-sensitive allele of the GAL4 repressor GAL80 ( $t u b-$ GAL80 ${ }^{T S}$ ) (McGuire et al., 2003) to restrict tau expression to adult glia. The GAL80 system is temperature dependent, with significant repression of transgene expression at $17^{\circ} \mathrm{C}$, moderate repression at $25^{\circ} \mathrm{C}$, and minimal repression at $30^{\circ} \mathrm{C}$ (supplemental Fig. S1, available at www.jneurosci.org as supplemental material).

We performed lifespan analyses to evaluate the toxicity of human tau in adult fly glial cells. We observed a marked reduction in lifespan of glial tau transgenic flies compared with control flies (Fig. 1). A reduction in lifespan was also observed when glial tau transgenic and control flies were aged at $25^{\circ} \mathrm{C}$, a more typical laboratory culture temperature (supplemental Fig. S2, available at www.jneurosci.org as supplemental material). Because $G A L 80^{T S}$ produced moderate repression of tau expression at the lower temperature $\left(25^{\circ} \mathrm{C}\right.$ ) (supplemental Fig. S1, available at www.jneurosci.org as supplemental material), the effect of tau on decreasing lifespan was more modest than that seen in Figure 1.

\section{Cell-autonomous and non-cell-autonomous death in glial tauopathy model}

To determine the extent to which cell death accompanied the reduction in lifespan, we assayed aged control and glial tau transgenic flies for the presence of TUNEL-positive nuclei. TUNEL marks fragmented DNA within nuclei of apoptotic cells and has been shown previously to identify dying cells in a Drosophila model of neuronal tauopathy (Khurana et al., 2006; Dias-Santagata et al., 2007). We performed TUNEL on 10-, 20-, and 30-d-old glial tau transgenic and control flies and observed a significant age-

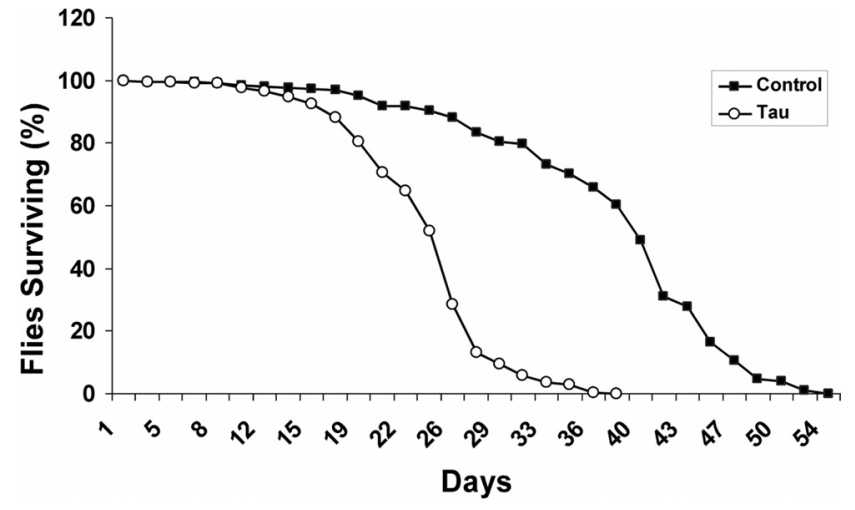

Figure 1. Expression of human tau in adult Drosophila glia reduces lifespan. Fly crosses were performed at $17^{\circ} \mathrm{C}$ to minimize transgene expression using the temperature-dependent repressor $G A L 80^{T S}$ during development. During eclosion, adult glial tau transgenic (open circles) and control (filled boxes) flies were aged at $30^{\circ} \mathrm{C}$ to derepress transgenic tau expression. A marked reduction in lifespan of glial tau transgenic flies is observed compared with control flies. Genotype of tau transgenic flies: UAS-tau ${ }^{W T} /$ repo-GAL4 tub-GAL8O ${ }^{T S}$. Genotype of control flies: repo-GAL4 tub-GAL8O $\%$.

dependent increase in TUNEL-positive cells in 20- and 30-d-old glial tau transgenic flies (Fig. $2 \mathrm{~A}$ ).

We performed fluorescent TUNEL and immunofluorescence using an antibody against the glial-specific marker repo (Lai and Lee, 2006; Ziegenfuss et al., 2008) to determine the identity of apoptotic cells observed in aged glial tau transgenic flies. Analysis of 30-d-old glial tau transgenic flies revealed that TUNEL positivity colocalized with repo-positive glial nuclei and was essentially absent in control flies (Fig. 2A,B) (supplemental Fig. S3, available at www.jneurosci.org as supplemental material). To further explore the mechanism of glial cell death, we performed double-labeled immunofluorescence using antibodies against repo and activated caspase, an upstream effector of apoptosis. We observed the presence of activated caspase staining within the cytoplasm of repo-positive glial cells in glial tau transgenic flies (Fig. 2C), supporting an apoptotic mechanism of cell death.

To determine whether the expression of human tau in fly glial cells resulted in non-cell-autonomous toxic effects on neurons, we performed fluorescent TUNEL and immunofluorescence using an antibody against the cholinergic neuronal marker ChAT on 30-d-old flies. ChAT is a cytoplasmic marker and in Drosophila neurons labels the thin rim of cytoplasm surrounding cholinergic neuronal nuclei (Fig. 2D). Analysis of 30-d-old glial tau transgenic flies revealed the presence of ChAT staining in neurons containing TUNEL-positive nuclei (Fig. 2D) establishing that non-cell-autonomous neuronal death is a feature of aged glial tau transgenic flies. Colocalization of activated caspase- 3 and ChAT confirmed cholinergic neuron apoptotic cell death in 30-d-old glial tau transgenic flies (Fig. 2E). We were unable to assign glial or neuronal identity to all TUNEL-positive cells because the cell-type-specific markers available to us were lost in many apoptotic cells.

\section{Sarkosyl-insoluble, phosphorylated tau accumulates in Drosophila glial cells}

The accumulation of Sarkosyl-insoluble species of tau is a defining characteristic of tau isolated from brains of patients with Alzheimer's disease and related tauopathies (Kosik et al., 1986; Ksiezak-Reding et al., 1994). To determine whether expression of human tau in Drosophila glial cells resulted in the formation of similar species of tau, we performed Sarkosyl extractions on fly 
A

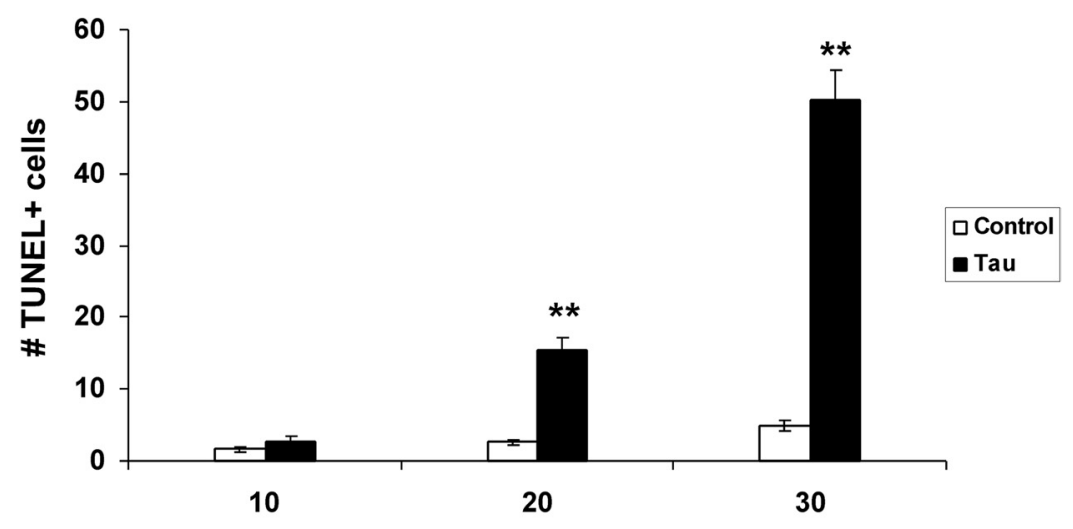

Age (Days)
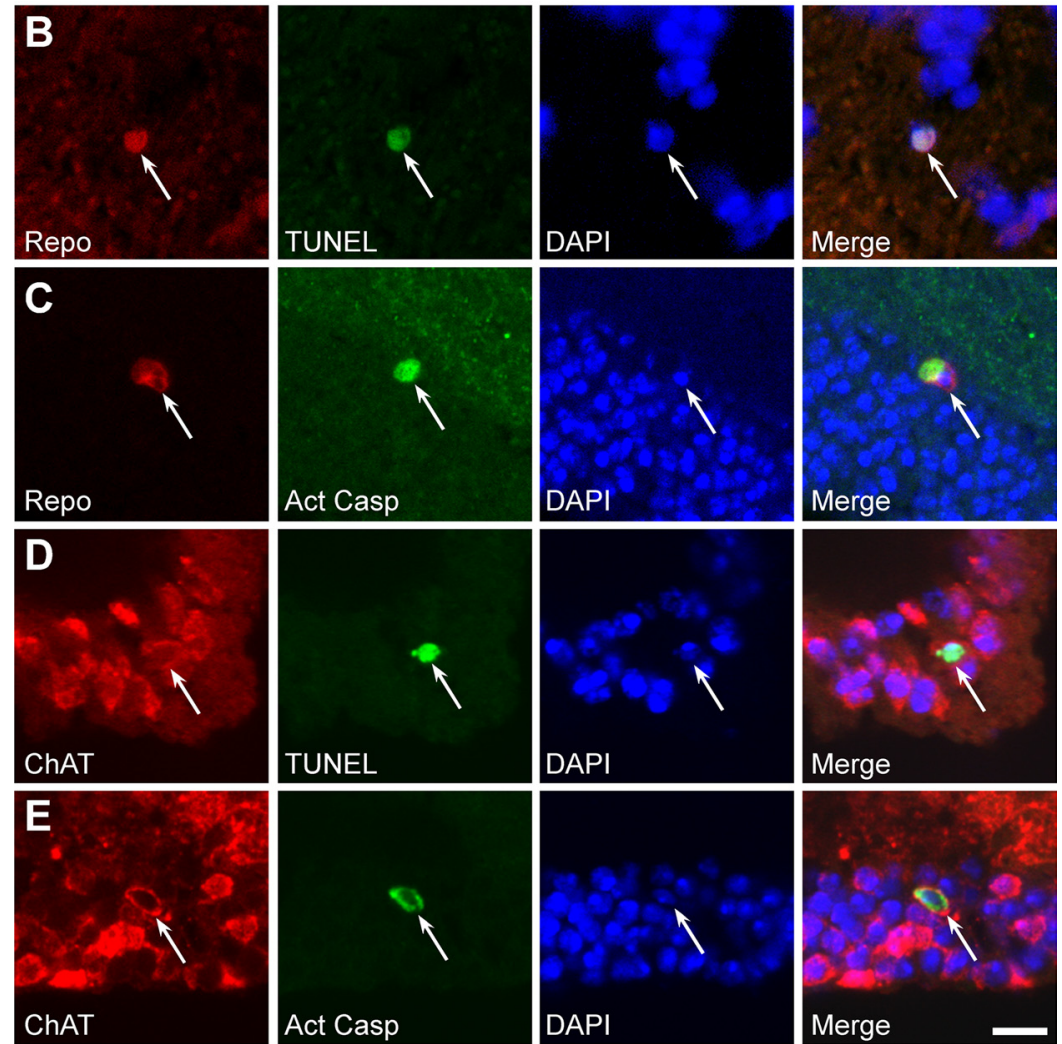

Figure 2. Age-dependent apoptotic glial and neuronal cell death in glial tau transgenic flies. $A$, Quantification of the number of TUNEL-positive cells in frontal brain sections throughout the entire brain of glial tau transgenic (black) and control (white) flies. At least 12 hemibrains of 10-, 20, and 30-d-old control and glial tau transgenic flies were analyzed. A statistically significant increase in the number of TUNEL-positive nuclei is observed in 20- and 30-d-old glial tau transgenic flies compared with control flies. Statistical analysis was performed with an unpaired $t$ test. ${ }^{* *} p<0.01$. Error bars represent \pm SEM. $\boldsymbol{B}-\boldsymbol{E}$, Double-label immunofluorescence and TUNEL on frontal sections of 30-d-old glial tau transgenic flies. Colocalization of the glial-specific protein repo (red; $\boldsymbol{B}, \boldsymbol{C}$ ) with TUNEL-positive nuclei (green, $\boldsymbol{B}$ ) and activated caspase-3 (Act Casp; green, $\boldsymbol{C}$ ) reveals the presence of apoptotic glial nuclei in aged glial tau transgenic flies (arrows, B, C). Double-label immun ofluorescence with the cytoplasmic cholinergic neurona marker ChAT (red) and TUNEL labeling (green) identifies cytoplasm of a cholinergic neuron surrounding a TUNEL-positive nucleus (arrows, D), demonstrating cholinergic neuronal cell death in aged glial tau transgenic flies. Apoptotic cholinergic neuronal cell death was further confirmed by colocalization of ChAT staining (red) and activated caspase-3 (green) in glial tau transgenic flies (arrows, E). Nucleiare counterstained with 4', 6' -diamidino-2-phenylindole (DAPI) (blue). Scale bar, $5 \mu \mathrm{m}$. Genotype of tau transgenic flies: UAS-tau ${ }^{W T} / \mathrm{repo}^{-}$ GAL4 tub-GAL8OTS . Genotype of control flies: repo-GAL4 tub-GAL $80^{T S} /+$. Flies were aged at $30^{\circ} \mathrm{C}$.

head homogenates from 1-, 10-, and 20-d-old glial tau transgenic flies and 20-d-old control flies (Fig. 3A). As expected, we did not detect the presence of tau in homogenates of 1-d-old glial tau transgenic flies grown at the restrictive $17^{\circ} \mathrm{C}$ temperature (Fig. $3 A, \mathrm{~d} 1)$. We did observe an age-dependent increase in the amount of Sarkosyl-insoluble tau recovered between days 10 and 20 (Fig. 3A, right), whereas levels of Sarkosyl-soluble tau were similar at the two time points (Fig. 3A, left).

To determine the ultrastructural characteristics of tau present in the Sarkosylinsoluble fraction, we performed immunoelectron microscopic analysis on the Sarkosyl-insoluble fraction isolated from 20-d-old glial tau transgenic and control flies, using a phosphorylation-independent polyclonal anti-tau antibody (Dako). We identified tau-immunoreactive straight filaments in aged glial tau transgenic flies (Fig. 3B) (supplemental Fig. S4A, available at www.jneurosci.org as supplemental material). Similar fibrils were not detected in the Sarkosyl-insoluble fraction derived from control flies. These studies demonstrate that tau isolated from glial tau transgenic flies forms Sarkosyl-insoluble fibrils, a defining characteristic of pathological tau derived from human tauopathy brains (KsiezakReding et al., 1994).

The presence of abnormally phosphorylated tau is also a prominent feature of human tauopathies (Ballatore et al., 2007). To determine the phosphorylation state of human tau expressed in adult Drosophila glial cells, we performed Western blot analysis on fly head homogenates and compared the electrophoretic mobility with and without the presence of lambda phosphatase (Fig. 3C). A pronounced phosphatase-dependent shift in electrophoretic mobility was observed in samples from 10- and 30-d-old glial tau transgenic flies, suggesting that human tau is phosphorylated when expressed in Drosophila glial cells.

Phosphorylation of tau at particular sites can be identified using specific antibodies. A number of these phosphorylation events are associated with human tauopathies (Buée et al., 2000). To determine whether these tauopathy-associated sites were phosphorylated in our Drosophila model of glial tauopathy, we performed Western blot analysis of fly head homogenates using a number of phosphospecific antibodies. Western blots of 1-, 10-, and 30-d-old glial tau transgenic flies and 30-d-old control flies revealed that glial-expressed tau is phosphorylated at the disease-associated phosphorylation sites AT8, AT100, AT180, and AT270 (Fig. $3 D)$. As expected, no signal was detected in 30-d-old control flies and 1-d-old glial tau transgenic flies. Phosphorylation at the AT8 and AT100 epitopes increased with age, whereas AT180 and AT270 levels remained at constant levels from days 10 to 30 . These data suggest that kinases resident in fly glial cells are capable of phosphorylating tau, similar to observations 
made when human tau was expressed in Drosophila neurons (Wittmann et al., 2001; Jackson et al., 2002; Steinhilb et al., 2007a).

Expression of human tau in Drosophila glial cells results in glial fibrillary tangle formation

Neurofibrillary and glial fibrillary tangles are pathological hallmarks of tauopathies and can be identified using a variety of histochemical and immunohistochemical staining techniques (Chin and Goldman, 1996; Ballatore et al., 2007). To determine whether similar inclusion pathology was present in glial tau transgenic flies, we performed immunohistochemistry on paraffin-embedded sections of 30-d-old glial tau transgenic and control brains using antibodies against disease-associated phosphoepitopes of tau. Low-magnification images of sections stained with the AT100 monoclonal antibody show punctate immunoreactivity throughout the cortex and neuropil of 30-d-old glial tau transgenic flies (Fig. 4A, arrows), with no detectable immunostaining observed in age-matched control flies (Fig. 4B). Higher-magnification images of AT100 immunostaining reveal inclusion bodies in glial tau transgenic flies (Fig. 4C, arrows) and no detectable inclusions in age-matched control flies (Fig. 4D). Inclusion bodies were also recognized by other phosphorylation- and conformation-specific antibodies, including AT8, antiPSer212, anti-PSer214, PHF1, MC1, and Alz50 (supplemental Fig. S4 B, C, available at www.jneurosci.org as supplemental material) (data not shown). We identified phosphorylated tau in the proximal processes of glial cells (Fig. $4 E, F$ ) and also in the distal processes of glial cells (Fig. $4 G$ ), similar to findings in progressive supranuclear palsy and corticobasal degeneration (Feany and Dickson, 1996). Double-labeled immunofluorescence using antibodies against repo and tau phosphorylated at serine 214 confirmed the glial identity of these tauexpressing cells (Fig. $4 \mathrm{H}$, arrow) and identified phosphorylated tau in glial processes (Fig. $4 H$, arrowhead).

To further explore the similarity of inclusions in our glial tau transgenic flies to authentic fibrillary tau inclusions formed in human diseases, we performed Bielschowsky silver stain and thioflavin-S staining on 30-d-old glial tau transgenic and control flies. Bielschowsky silver staining identified inclusions in glial tau transgenic flies (Fig. 4I, arrows) but not in control flies (Fig. 4J). Inclusions were also stained with thioflavin-S (Fig. $4 K$, arrows). No thioflavin-S stained structures were present in control flies (Fig. 4L).

Finally, to characterize the inclusions at the ultrastructural level, we performed transmission electron microscopy on sections of 25-d-old glial tau transgenic and age-matched control fly brains. Fibrillar aggregates were present in tau transgenic but not control flies (Fig. 4M) (data not shown). These aggregates were composed of straight filaments with diameter of 10-15 nm, similar to the glial fibrillary tangles found in tauopathy brains (Buée et al., 2000).
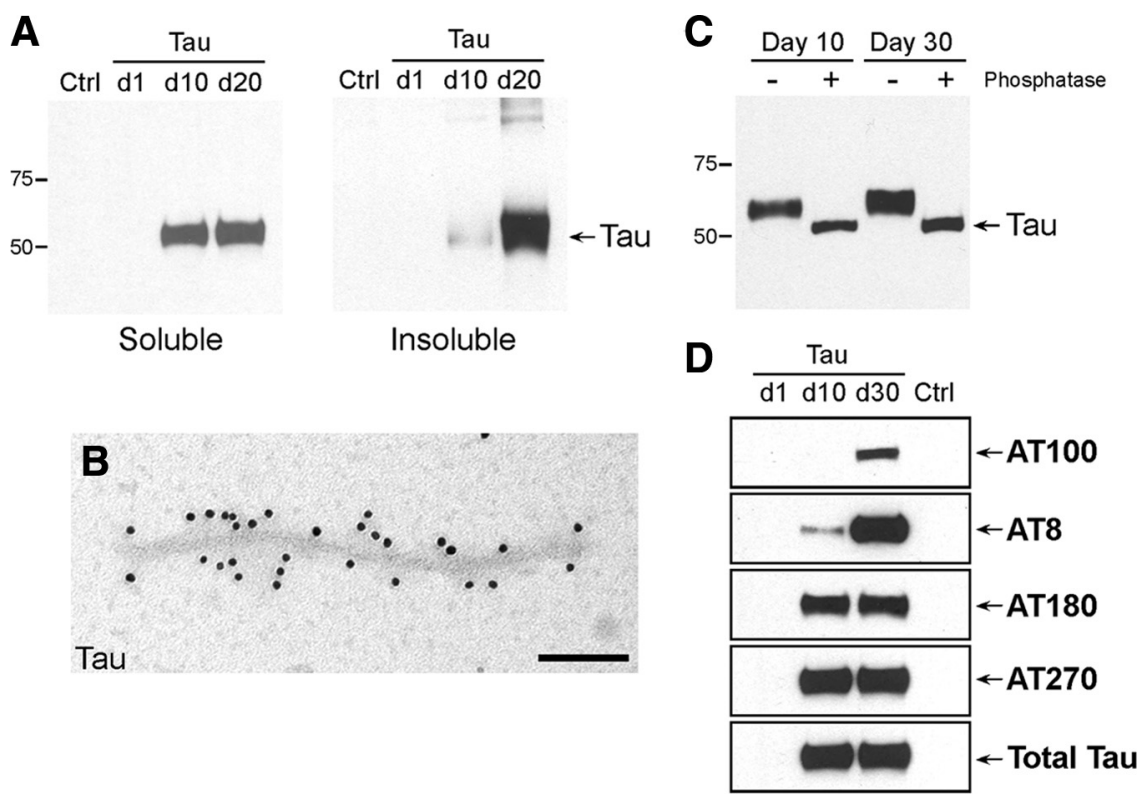

Figure 3. Insoluble, phosphorylated tau accumulates in aged glial tau transgenic flies. $\boldsymbol{A}$, Western blot analysis performed on -soluble and -insoluble fractions of homogenized control and glial tau transgenic flies. Sarkosyl-soluble tau is detected in

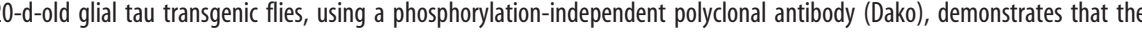
glial tau transgenic flies treated with or without lambda phosphatase. A phosphatase-dependent shift in electrophoretic mobility 作 (lies. Total tau was detected using a phosphorylation-independent polyclonal antibody (Dako). Genotype of tau transgenic flies: $U A S-$ tau $^{W T} /$ repo-GAL4 tub-GAL8O $0^{T S}$. Genotype of control flies: repo-GAL4 tub-GAL8OTS $/$. Flies were aged at $30^{\circ} \mathrm{C}$.

To confirm that these inclusions were composed of tau, we performed immunoelectron microscopy on 25-d-old control and glial tau transgenic flies using the phosphorylation-specific antibody AT8 and confirmed the presence of fibrillar tau inclusions in tau transgenic flies (Fig. $4 N$ ). In summary, we have used the following features to support our identification of inclusion bodies with important similarities to authentic tangles in brains from our glial tau transgenic flies: well demarcated morphology at the light and electron microscopic levels, reactivity with thioflavin-S and Bielschowsky silver stains, formation of fibrils with size and morphology similar to those seen in comparable four repeat human tauopathies, and localization within aggregates of abnormally phosphorylated and conformationally altered tau as evidenced by immunoreactivity with phosphorylation- and conformation-specific antibodies.

\section{Reducing tau expression prevents toxicity without changing inclusion number}

We took advantage of our regulated expression system to probe the effects of reducing tau levels after onset of toxicity and tangle formation. Fly crosses were performed at the restrictive $17^{\circ} \mathrm{C}$ temperature. After eclosion, animals were aged at $30^{\circ} \mathrm{C}$ for $20 \mathrm{~d}$ to allow tau expression, neurotoxicity, and inclusion deposition. Flies were then transferred to $17^{\circ} \mathrm{C}$ for $10 \mathrm{~d}$ to reduce tau expression. Separate groups of glial tau transgenic animals were aged to $30 \mathrm{~d}$ at a continuous temperature of $17^{\circ} \mathrm{C}$ or $30^{\circ} \mathrm{C}$. Tau was min- 

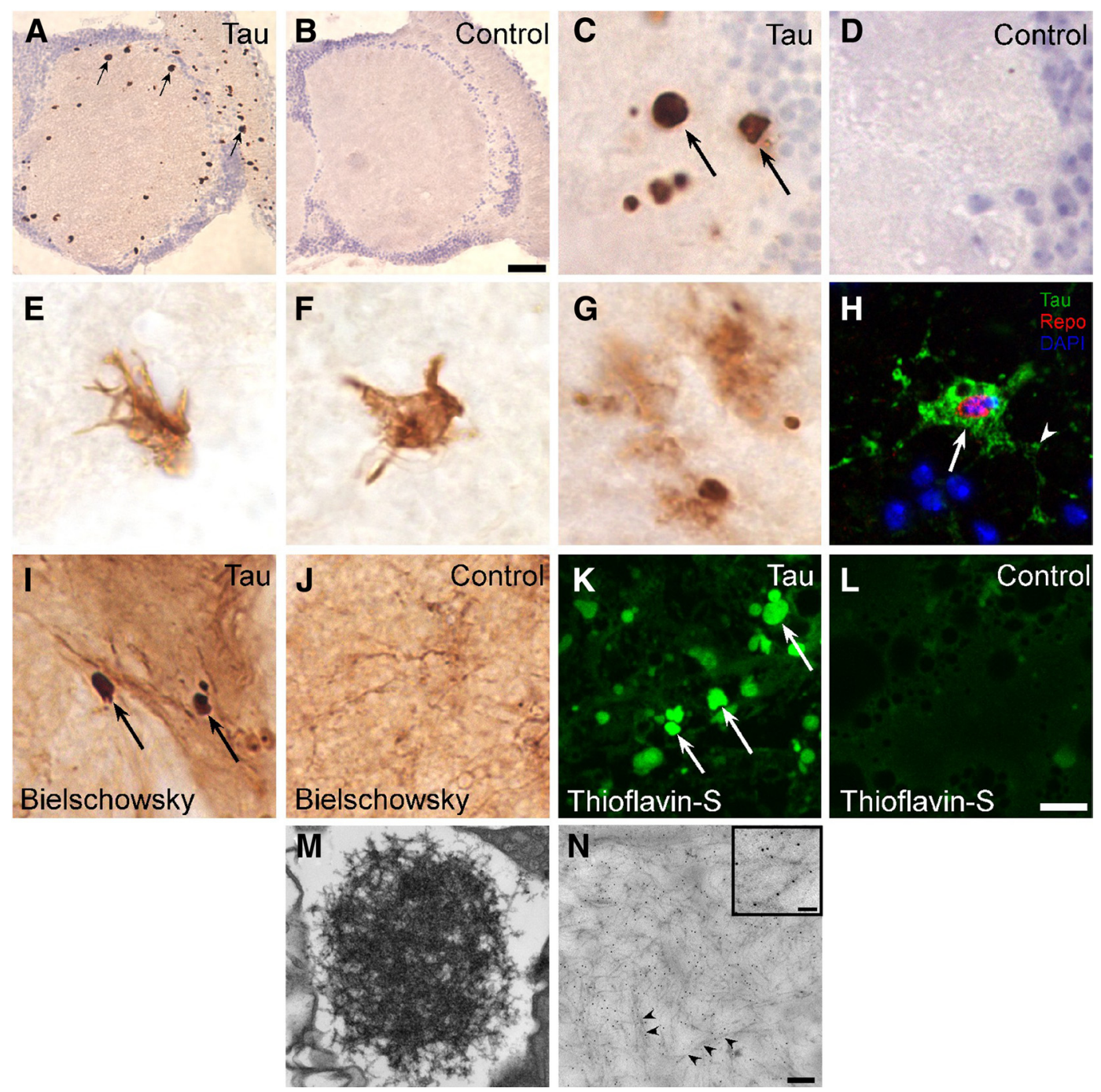

Figure 4. Glial fibrillary tangle formation in tau transgenic flies. $A-G$, Immunohistochemistry of frontal sections of 30-d-old glial tau transgenic and control flies using an antibody against the phosphorylation and conformation-specific antibody AT100. Low-magnification images of glial tau transgenic flies reveal numerous inclusions throughout the cortex and neuropil of the brain of glial tau transgenic flies (arrows, $\boldsymbol{A})$. There is no staining in control flies $(\boldsymbol{B}, \boldsymbol{D})$. $\mathbf{C}-\boldsymbol{G}$, High-magnification images reveal AT100-positive inclusion bodies $(\boldsymbol{C})$, tau-positive glial processes $(\boldsymbol{E}, \boldsymbol{F})$, and staining of distal processes in the neuropil (G). $\boldsymbol{H}$, Double-labeled immunofluorescence using antibodies against repo (red) and tau phosphorylated at residue 214 (green) confirms that phosphorylated tau is present in glial cell bodies (arrow, $\boldsymbol{H}$ ) and glial processes (arrowhead, $\boldsymbol{H}$ ). Nuclei are counterstained with DAPI (blue). $\boldsymbol{I}, \boldsymbol{J}$, Bielschowsky silver staining of glial tau transgenic $(\boldsymbol{I})$ and control flies $(\boldsymbol{J})$ stains inclusions in glial tau transgenic flies (arrows, $\boldsymbol{I}) . \boldsymbol{K}, \boldsymbol{L}$, Thioflavin-S staining of glial tau transgenic $(\boldsymbol{K})$ and control $(\boldsymbol{L})$ flies identifies inclusions in glial tau transgenic flies (arrows, $\boldsymbol{K}$ ). $\boldsymbol{M}$, Standard transmission electron microscopy of 25 -d-old glial tau transgenic flies reveals the presence of tau filaments. $N$, Immunoelectron microscopy using the phospho-specific antibody AT8 labels tau fibrils in glial tau transgenic flies. Inset in $\boldsymbol{N}$ displays higher magnification of tau-immunopositive fibrils. Scale bars: $A, B, 25 \mu \mathrm{m} ; C-L, 5 \mu \mathrm{m} ; \boldsymbol{M}, \boldsymbol{N}, 200 \mathrm{~nm}$; inset in $\boldsymbol{N}, 50 \mathrm{~nm}$. Genotype of tau transgenic flies: UAS-tau ${ }^{W T} /$ repo-GAL4 tub-GAL8O $0^{T S}$. Genotype of control flies: repo-GAL4 tub-GAL8O $/ 5$. Flies were aged at $30^{\circ} \mathrm{C}$.

imally expressed in flies aged at $17^{\circ} \mathrm{C}$ for the entire experimental period (Fig. $5 \mathrm{~A}$ ). As expected, aging flies at $30^{\circ} \mathrm{C}$ produced substantial tau expression. Reducing the culture temperature to $17^{\circ} \mathrm{C}$ for $10 \mathrm{~d}$ reduced tau levels compared with continuous aging at $30^{\circ} \mathrm{C}$ (Fig. $5 \mathrm{~A}$ ). A modest reduction in tau levels in the flies switched to $17^{\circ} \mathrm{C}$ for $10 \mathrm{~d}$ prevented cell death as assessed by TUNEL staining (Fig. 5B). In contrast, no alteration in the number of glial fibrillary tangles was observed after switching flies to $17^{\circ} \mathrm{C}$ for $10 \mathrm{~d}$ (Fig. $5 C, D$ ).

\section{STAT activity is reduced in glial tau transgenic flies}

To probe the mechanisms underlying the toxicity of human tau in Drosophila glial cells, we performed a deficiency screen designed to allow identification of enhancers and suppressors of glial tau toxicity and recovered suppressor of cytokine signaling $36 \mathrm{E}$ (Socs36E) in one of the candidate intervals (our unpublished results). SOCS proteins are inhibitors of JAK/STAT signaling in mammals and have been shown to perform similar functions in Drosophila (Callus and Mathey-Prevot, 2002; Yoshimura et al., 2007). Transgenic STAT-GFP reporter flies have been developed previously and characterized as indicators of STAT activity (Baeg et al., 2005; Bach et al., 2007). To determine whether STAT-GFP reporter activity was altered in glial tau transgenic flies, we performed Western blot analysis on 10-, 20-, and 30-d-old glial tau transgenic and control flies carrying the STAT-GFP reporter construct (Fig. 6A) (supplemental Fig. S5, available at www. jneurosci.org as supplemental material). We observed a decrease in STAT-GFP reporter activity in 20- and 30-d-old glial tau transgenic flies compared with control flies. To confirm that the decrease in STAT-GFP reporter activity was localized to glial cells, we performed double-labeled immunofluorescence on 30d-old glial tau transgenic and control flies using antibodies 
against the glial-specific proteins repo and GFP. We observed that STAT-GFP reporter activity was specifically reduced in the glial cells of glial tau transgenic flies compared with control flies (Fig. 6B). The observed decrease in STAT-GFP activity is unlikely to be attributable to substantial death of glial cells because nuclei of STAT-GFP-negative glial cells are observed in normal distributions in the fly brain (Fig. $6 \mathrm{~B}$, bottom row). Moreover, overexpression of polyglutamine expanded mutant human ataxin-3, which results in lethality of adult flies within $10 \mathrm{~d}$ after eclosion (driver: repo-GAL4,tub-GAL80 ${ }^{T S}$ ) does not affect STAT-GFP reporter activity (supplemental Fig. S6, available at www. jneurosci.org as supplemental material). These studies reveal that the expression of human tau in Drosophila glial cells results in a reduction of STAT reporter activity.

\section{JAK/STAT signaling mediates toxicity} To explore the role JAK/STAT signaling plays in mediating the toxicity associated with expression of tau in glial cells, we performed genetic experiments to manipulate key components of the JAK/STAT signaling pathway in glial cells. To determine whether the restoration of STAT activity could suppress toxicity, we increased levels of STAT using a UAS-STAT transgene (Brown et al., 2006). Overexpression of STAT significantly suppressed the number of TUNEL-positive cells in 30-d-old glial tau transgenic flies (Fig. $7 A$ ). Conversely, reducing the dosage of hopscotch (hop), the Drosophila homolog of JAK (Perrimon and Mahowald, 1986), in glial tau transgenic flies produced a significant increase in the number of TUNEL-positive cells in 30-d-old glial tau transgenic flies (Fig. 7A). Reduction of hop levels in control flies did not result in toxicity (Fig. $7 A$ )

We then altered levels of Socs36E, an inhibitor of JAK/STAT signaling, and assayed the effects on toxicity in glial tau transgenic animals. Decreasing Socs36E using a transgenic RNA interference (RNAi) construct significantly decreased toxicity, whereas overexpression of Socs36E suppressed toxicity (Fig. 7A). Decreasing Socs36E levels in the absence of tau was not toxic (Fig. 7A).

To confirm alterations in STAT activity with genetic manipulations of the JAK/STAT pathway that modified glial tau toxicity, we assayed STAT reporter activity in the modified genotypes. Quantitative analysis revealed an approximate sixfold decrease in STAT reporter activity in glial tau transgenic flies compared with control flies (Fig. 7B). RNAi-mediated knockdown of Socs36E significantly restored STAT reporter activity (Fig. 7B), and, conversely, overexpression of Socs $36 \mathrm{E}$ and reduction in hop levels both significantly enhanced the reduction in STAT reporter activity (Fig. 7B). We did not see consistent effects of JAK/STAT pathway modifiers on the phosphorylation of tau using the AT8, AT270, and AT100 antibodies, consistent with a role for the JAK/STAT pathway downstream of tau phosphorylation (data not shown).

Our results thus far demonstrate that expression of human tau
C

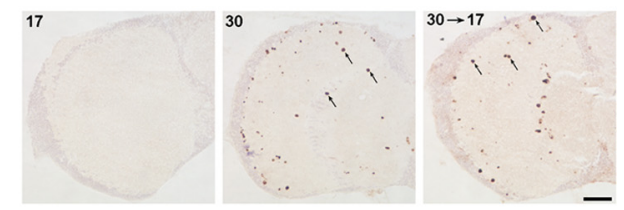

D

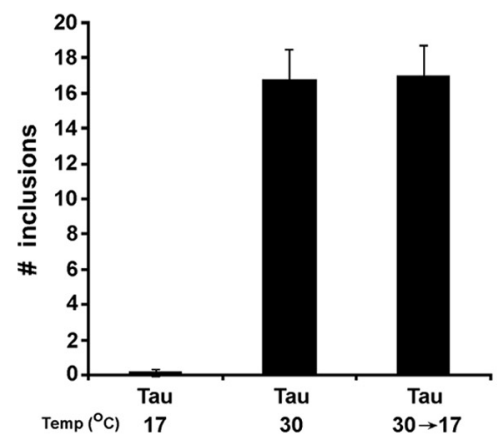

Figure 5. Reducing tau expression prevents toxicity without changing tangle burden. $\boldsymbol{A}$, Western blot analysis of tau levels in 列 列 (inclusions $>3 \mu \mathrm{m}$ in diameter were counted in a section of the medulla (as illustrated in $\mathbf{C}$ ) from six animals per genotype and temperature regimen. Statistical analysis was performed with one-way ANOVA with Student-Newman-Keuls. ${ }^{* *} p<0.01$. Error bars represent \pm SEM. Genotype of tau transgenic flies (Tau): UAS-tau ${ }^{W T} /$ repo-GAL4 tub-GAL80 ${ }^{T S}$. Genotype of control $(\boldsymbol{A}, \boldsymbol{B})$ flies: repo-GAL4 tub-GAL80 $\mathrm{TS} /+$

in glial cells produces death of glia and non-cell-autonomous neuronal death accompanied by glial fibrillary tangle formation and reduction in JAK/STAT pathway activation. However, in human tauopathies such as progressive supranuclear palsy, corticobasal degeneration, and related disorders, tau is present in both neurons and glia. We described previously a neuronal tauopathy model based on expression of wild-type human tau (Wittmann et al., 2001) in Drosophila neurons using the pan-neural driver elavGAL4. To determine whether neuronal and glial tau can interact in an in vivo animal model system, we expressed wild-type human tau in both neurons and glia. We aged the animals at $25^{\circ} \mathrm{C}$ to decrease tau expression with the $G A L 80^{T S}$ allele and thus minimize the toxicity of human tau expressed solely in neurons or glia. Remarkably, we found that, under conditions in which neither neuronal nor glial tau expression produced significant cellular toxicity as measured by the number of apoptotic cells, there was a striking increase in toxicity with combined expression of tau in both neurons and glia (Fig. 8). Phosphorylation of tau was only modestly increased in flies expressing tau in both neurons and glia (supplemental Fig. S7, available at www.jneurosci.org as supplemental material).

\section{Discussion}

In human tauopathies, the presence and detailed morphology of glial inclusions can be quite important diagnostically (Feany and Dickson, 1996; Berry et al., 2001); however, the significance of the accumulation of fibrillar tau in glial cells to the neurodegeneration characteristic of these disorders has been unclear. Patho- 
A
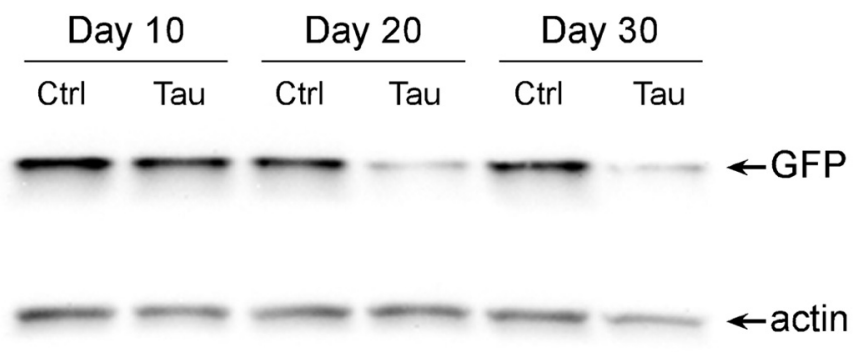

B
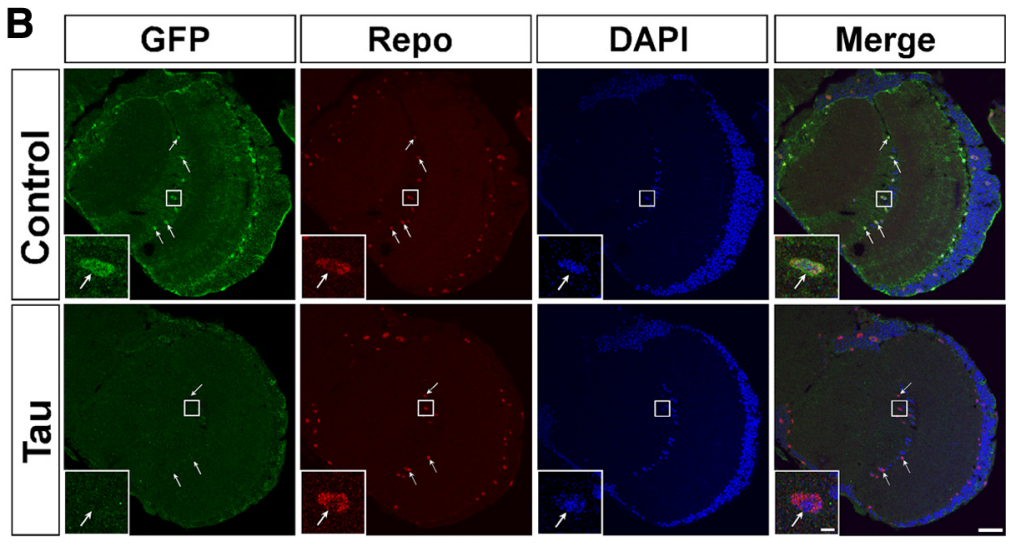

Figure 6. STAT-GFP reporter activity is decreased in glial cells of aged glial tau transgenic flies. $A$, Western blot analysis of STAT-GFP reporter levels in 10-, 20-, and 30-d-old STAT-GFP control (Ctrl) and glial tau transgenic (Tau) flies. A decrease in levels of GFP can be detected in 20- and 30-d-old glial tau transgenic flies compared with control flies (top). Actin levels are shown to illustrate equivalent protein loading (bottom). A representative blot illustrating the results of five independent experiments is shown. $\boldsymbol{B}$, Double-labeled immunofluorescence of frontal brain sections of 30-d-old glial tau transgenic and control flies containing the STAT-GFP reporter. STAT-GFP (green) is present in repo-positive glial cells (red) in control flies (top row). A reduction in STAT-GFP (green) is observed in repo-positive glial cells (red) in glial tau transgenic flies (bottom row). DAPI, $4^{\prime}, 6^{\prime}$-Diamidino2-phenylindole. Insets in $\boldsymbol{B}$-I are high-magnification images of individual glial cells outlined by white boxes in low-magnification images. Scale bar: $\boldsymbol{B}, 20 \mu \mathrm{m}$; insets, $2 \mu \mathrm{m}$. Genotype of tau transgenic flies (Tau): 10XSTAT-GFP/+; UAS-tau ${ }^{\text {WT }}$ repo-GAL4 tub-GAL8O $80^{T S} /+$. Genotype of control flies (Ctrl): 10XSTAT-GFP/+; repo-GAL4 tub-GAL8OSS + . Flies were aged at $30^{\circ} \mathrm{C}$.

logical analysis of tissue from patients with progressive supranuclear palsy suggested that glial tau inclusions represent a degenerative, rather than reactive, change based on lack of increased glial fibrillary acidic protein (GFAP) in affected astrocytes (Togo and Dickson, 2002). A positive correlation between neurofibrillary tangles and glial tau inclusions has been reported in the central gray matter, pontine nuclei, and tegmentum, which are plausible anatomic mediators of the major clinical symptoms in progressive supranuclear palsy (Ito et al., 2008). Furthermore, the glial-specific intermediate filament GFAP is a major target of oxidative damage in both the sporadic tauopathy Pick's disease (Muntane et al., 2006) and the genetic tauopathy FTDP-17 (Martinez et al., 2008). To establish an experimental system to explore the potential for glial tau to influence neurodegeneration, we created a model of glial tauopathy in the genetically powerful model system Drosophila. Our results demonstrate toxicity of wild-type human tau to glial cells and non-cell-autonomous death of neurons. Thus, our data support a role for glial tau in contributing to neurodegeneration in tauopathies. These findings are consistent with data from murine tauopathy models (Forman et al., 2005; Higuchi et al., 2005).

The toxicity associated with the Drosophila model of glial tauopathy is accompanied by the accumulation of inclusions similar at the light and electron microscopic levels to authentic human glial fibrillary tangles. The presence of numerous fibrillary tau tangles in our glial tauopathy model contrasts with the Drosophila model of neuronal tauopathy, in which neurodegen- eration occurs in the absence of significant neurofibrillary tangle formation (Wittmann et al., 2001). The reason for the discrepancy in tangle formation between the neuronal and glial tauopathy models is not clear. Fly kinases can phosphorylate human tau (Jackson et al., 2002; Nishimura et al., 2004; Steinhilb et al., 2007b), and coexpression of Shaggy, the Drosophila glycogen synthase kinase-3 homolog, induces neurofibrillary-type changes in flies expressing tau in neurons (Jackson et al., 2002). Thus, the disparity in tangle formation between the neuronal and glial Drosophila models of tauopathy might reflect altered kinase and phosphatase levels in fly glia compared with neurons. However, although there is substantial phosphorylation of glial tau (Fig. 3), a direct comparison of phosphorylation in neurons and glia does not suggest increased phosphorylation of glial tau, at least at the sites examined (supplemental Fig. S7, available at www. jneurosci.org as supplemental material). Rather, an increase in total levels of tau may drive tangle formation in glia (supplemental Fig. S7, available at www. jneurosci.org as supplemental material). Alternatively, other aspects of the glial cellular milieu might promote tangle formation. In mice, endogenous mouse tau inhibits the formation of neuronal tangles by transgenically expressed human tau (Andorfer et al., 2003). Because fly tau is expressed in neurons (Heidary and Fortini, 2001), the presence of fly tau could similarly inhibit the formation of neuronal tangles.

Inclusions appear in many neurodegenerative diseases, but the role these aggregates play in pathogenesis remains a subject of debate. Studies using repressible neuronal tau transgenic mice have demonstrated a dissociation of neurofibrillary tangle formation and cell death (Santacruz et al., 2005; Spires et al., 2006). In contrast, studies in mouse models of Huntington's disease have suggested that inclusions found in these models are capable of sequestering key transcription factors, which contributes to pathology (Yamanaka et al., 2008). In a first exploration of the relationship between tangle formation and toxicity in our model, we have reduced tau expression after the onset of cellular toxicity and inclusion deposition. We find that even a modest reduction in the levels of tau markedly reduces neuronal and glial cell death, without altering the number of tangles (Fig. 5). These findings are similar to those of Santacruz et al. (2005) in a repressible neuronal murine model of tauopathy. The significant remaining tau protein after $10 \mathrm{~d}$ at the restrictive temperature for tau expression in our animals (Fig. 5A) is also consistent with previous studies suggesting a very long half-life for tau in vivo (Mercken et al., 1995; Morales-Corraliza et al., 2009).

Long thought to be passive structural elements in the brain, glia are now being appreciated for their active participation in processes such as synapse development, neurotransmitter buffering, and response to injury and disease. Thus, neural-glial interactions appear to be essential for proper brain functioning and 

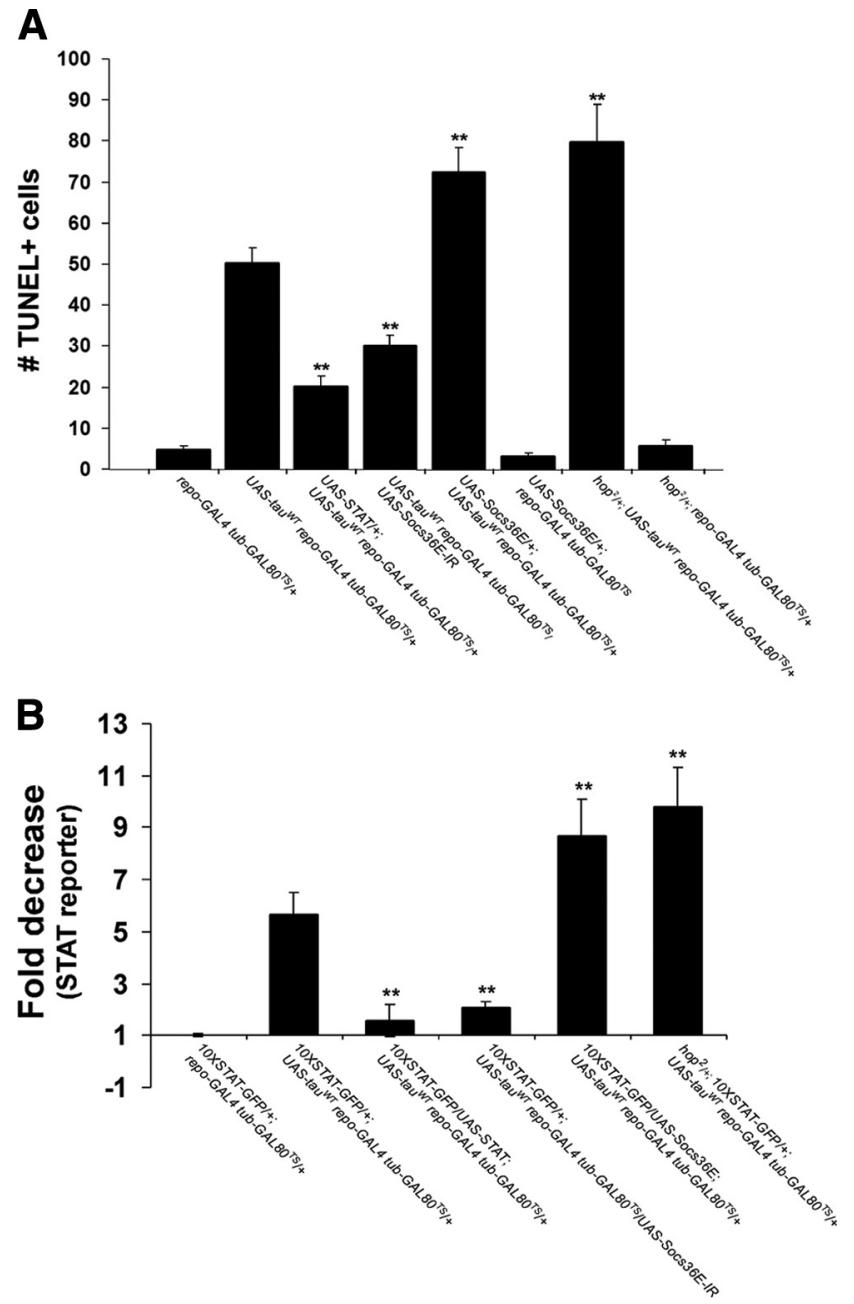

Figure 7. Genetic manipulation of JAK/STAT signaling modifies toxicity and STAT-GFP reporter activity in glial tau transgenic flies. $A$, Quantification of the number of TUNELpositive cells of 30-d-old glial tau transgenic flies alone (genotype: UAS-tau ${ }^{W T} /$ repo- $^{-}$ GAL4 tub-GAL8OTS ) or with genetic manipulation of JAK/STAT signaling components. Tau toxicity is suppressed by coexpression of a STAT transgene (UAS-STAT/+; UAS-tau ${ }^{\text {WT }}$ repo-GAL4 tub-GAL8OSS $/+$ ) and RNAi-mediated knockdown of Socs36E (UAS-tau ${ }^{\text {WT }}$ repo-GAL4 tub-GAL8OTS/UAS-Socs36E-IR) and enhanced by overexpression of Socs36E (UAS-Socs36E/+; UAS-tau ${ }^{\text {WT }}$ repo-GAL4 tub-GAL80 $\mathrm{TS} /+$ ) and $50 \%$ reduction in hop levels using the null allele, hop ${ }^{2}\left(h_{0}^{2} /+; U A S-t a u^{W T}\right.$ repo-GAL4 tub-GAL8O $/$ / $/$ ). No toxicity is observed in control flies that overexpress Socs36E (UAS-Socs36E/repo-GAL4 tub-GAL8O ${ }^{T S}$ ) or control flies with $50 \%$ reduction in hop levels (hop ${ }^{2} /+$; repo-GAL4 tub-GAL8O $/ \mathrm{TS}$ ). At least 12 hemibrains per genotype were analyzed. Statistical analysis was performed with one-way ANOVA with Student-Newman-Keuls test. ${ }^{* *} p<0.01$. Error bars represent \pm SEM. Genotype of control flies: repo-GAL4 tub-GAL8O $0^{T S} /+$. B , Densitometric analysis of Western blots measuring GFP levels. STAT-GFP levels in 30- $d$ old glial tau transgenic flies with genetic manipulation of JAK/STAT signaling components. The decrease in STAT-GFP observed in glial tau transgenic flies (10XSTAT-GFP/+; UAS-tau ${ }^{W T}$ repo-GAL4 tub-GAL80 8 / + ) compared with control flies (10XSTAT-GFP/+; repo-GAL4 tub-GAL8OTS $/+$ ) is significantly restored by coexpression of STAT (10XSTATGFP/UAS-STAT; UAS-tau ${ }^{W T}$ repo-GAL4 tub-GAL8O ${ }^{T S} /+$ ) and RNAi-mediated reduction of SOCS36E (10XSTAT-GFP/+; UAS-tau ${ }^{W T}$ repo-GAL4 tub-GAL8O $0^{\text {TS } / U A S-S O C S 36 E-I R) . ~}$ Reduction in STAT-GFP in glial tau transgenic flies is significantly enhanced by overexpression of Socs36E (UAS-SOCS36E/10XSTAT-GFP; UAS-tau ${ }^{W T}$ repo-GAL4 tub$\left.G A L 80^{T S} /+\right)$ or a $50 \%$ reduction in hop levels $\left(\right.$ hop $^{2} /+; 10 X S T A T-G F P /+;$ UAS-tau ${ }^{W T}$ repo-GAL4 tub-GAL8OSS $/+$ ). The results represent the average of at least six animals per genotype, in which protein homogenates were prepared and assayed separately from at least six individual fly heads per genotype. Statistical analysis was performed with oneway ANOVA with Student-Newman-Keuls test. ${ }^{* *} p<0.01$. Error bars represent \pm SEM. Flies were aged at $30^{\circ} \mathrm{C}$.

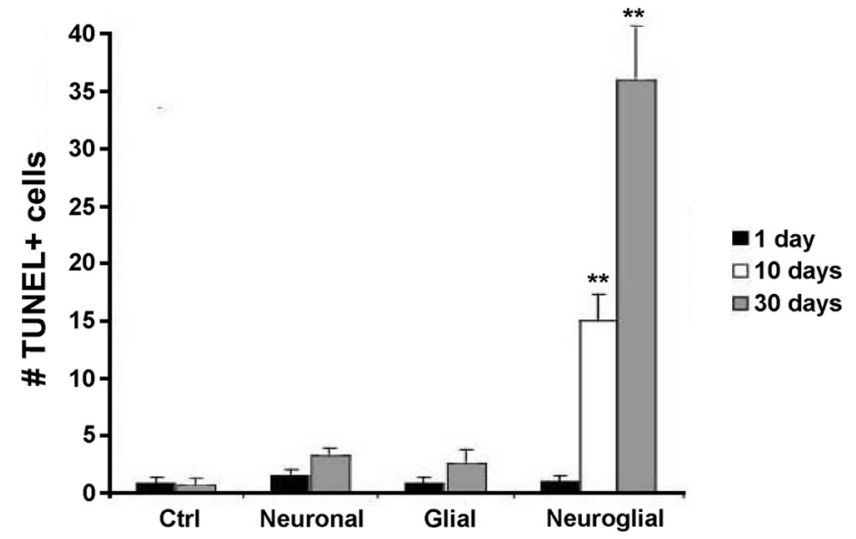

Figure 8. Synergistic toxicity of neuronal and glial tau. Quantification of the number of TUNEL-positive cells of 1-, 10-, and 30-d-old transgenic flies expressing tau in neurons (genotype: elav-GAL4/+;UAS-tau ${ }^{W T} /$ tub-GAL8O ${ }^{T S}$ ), glia (genotype: UAS-tau ${ }^{W T} / r e p o-G A L 4$ tub$G A L 80^{T S}$ ), or in both neurons and glia (genotype: elav-GAL4/+;UAS-tau ${ }^{W T}$ repo-GAL4 tub$G A L 80^{T S} /+$ ), and in control flies (genotype: elav-GAL4/+,repo-GAL4 tub-GAL8O ${ }^{T S} /+$ ). A statistically significant increase in the number of TUNEL-positive nuclei is observed in 10- and 30-d-old flies expressing tau in neurons and glia. Statistical analysis was performed with oneway ANOVA with Student-Newman-Keuls test. ${ }^{* *} p<0.01$. Error bars represent \pm SEM. Flies were aged at $25^{\circ} \mathrm{C}$ to support moderate expression of tau.

cell viability (Ransom et al., 2003; Allen and Barres, 2009). In Drosophila, glial cells are responsible for the phagocytic clearance of cellular debris in the adult fly (MacDonald et al., 2006), and targeted ablation of glial cells has been shown to result in the death of neuronal cells during fly development (Booth et al., 2000; Sepp et al., 2001). Additionally, fly mutants characterized by glial cell loss display secondary neuronal degeneration in adults (Xiong and Montell, 1995), underscoring the essential role of glial cells in the fly brain. Our studies further establish the importance of proper neural-glial interactions in the adult fly brain because glial-specific expression of tau resulted in both glial and non-cell-autonomous neuronal cell death (Fig. 2). Recent studies from conditional glial-derived neurotrophic factor knock-out mice demonstrate that neuronal viability in adult mice is also dependent on proper maintenance of glial trophic support (Pascual et al., 2008). Glial-secreted neurotrophic factors have yet to be identified in Drosophila, but the glial tau transgenic flies described in this study could potentially be useful in this search.

The ability to use genetic screening methods to identify cellular pathways and proteins that underlie disease pathogenesis is an important application of Drosophila models of human neurodegenerative disease (Muqit and Feany, 2002; Bonini and Fortini, 2003; Marsh and Thompson, 2006). In an initial approach to genetic screening in our glial tauopathy model, we have obtained genetic evidence strongly supporting a role for JAK/STAT signaling in mediating toxicity in our model (Fig. 7) using the number of dying cells as measured by TUNEL staining as a readout. An important caveat of the current work is the need for confirmation of the influence of JAK/STAT manipulation on the overall health of glial tau transgenic animals using additional assays. Using reporters for the JAK/STAT signaling pathway, we find a reduction in signaling in glial cells expressing tau. JAK/STAT signaling is a highly conserved signaling pathway that is involved in an array of diverse cellular processes and is central to the transduction of inflammatory signals. Alzheimer's disease and related tauopathies are characterized by gliosis and increased levels of inflammatory cytokines (Heneka and O'Banion, 2007). JAK/STAT 
signaling is activated as astrocytes become reactive in response to 1-methyl-4-phenyl-1,2,3,6-tetrahydropyridine toxicity and scrapie infection (Schwartz and Nishiyama, 1994; Sriram et al., 2004). Potential effectors of JAK/STAT signaling in astrocytes include regulation of astrocytic intercellular communication through modulation of connexin expression (Ozog et al., 2004), and putative downstream effectors have been implicated in glial glutamate uptake of stem-cell-derived astrocytes (Wilczynska et al., 2009). Of note, studies using a mouse model of glial tauopathy demonstrated that expression of tau in astrocytes leads to an impairment of glutamate transport (Dabir et al., 2006). Thus, investigation of JAK/STAT signaling is a promising candidate for additional evaluation in human tauopathies.

In this study, we reveal a synergistic increase in toxicity with coexpression of tau in both neurons and glial cells. When tau was expressed at modest levels, which did not result in significant cell death in neurons or glial cells alone, a significant increase in toxicity was revealed during coexpression of tau in both cell types (Fig. 8). These findings are noteworthy because human tauopathies, including progressive supranuclear palsy, corticobasal degeneration, and Pick's disease, are characterized by the presence of fibrillar tau inclusions in both cell types (Feany and Dickson, 1996; Berry et al., 2001). Because many biological interactions may be nonlinear, a synergistic interaction between neuronally and glially expressed tau may not necessarily be surprising; however, our findings do suggest that targeting glial tau may have a significant impact therapeutically. Because glial tau accumulation has been reported in Alzheimer's disease (Papasozomenos, 1989a,b; Nakano et al., 1992; Nishimura et al., 1995) and even in normal aging as accumulation of significant numbers of thornshaped astrocytes (Schultz et al., 2004), our model could have implications beyond traditionally defined glial tauopathies such as progressive supranuclear palsy and corticobasal degeneration.

In summary, we report a Drosophila model of glial tauopathy that recapitulates important features of the human disorders, including formation of glial fibrillary tangles. Toxicity is mediated through reduced JAK/STAT signaling in glial cells and noncell-autonomous neuronal degeneration. Our model represents a genetically tractable tool in which to continue to dissect the mechanisms of glial tau toxicity. Furthermore, our work suggests that preventing glial accumulation and expression of tau in human tauopathies may be a useful therapeutic strategy, particularly because we demonstrate a striking enhancement in toxicity with expression of tau in both neurons and glia, as occurs in many tauopathies.

\section{References}

Allen NJ, Barres BA (2009) Neuroscience: glia-more than just brain glue. Nature 457:675-677.

Andorfer C, Kress Y, Espinoza M, de Silva R, Tucker KL, Barde YA, Duff K, Davies P (2003) Hyperphosphorylation and aggregation of tau in mice expressing normal human tau isoforms. J Neurochem 86:582-590.

Auluck PK, Chan HY, Trojanowski JQ, Lee VM, Bonini NM (2002) Chaperone suppression of alpha-synuclein toxicity in a Drosophila model for Parkinson's disease. Science 295:865-868.

Bach EA, Ekas LA, Ayala-Camargo A, Flaherty MS, Lee H, Perrimon N, Baeg GH (2007) GFP reporters detect the activation of the Drosophila JAK/ STAT pathway in vivo. Gene Expr Patterns 7:323-331.

Baeg GH, Zhou R, Perrimon N (2005) Genome-wide RNAi analysis of JAK/ STAT signaling components in Drosophila. Genes Dev 19:1861-1870.

Ballatore C, Lee VM, Trojanowski JQ (2007) Tau-mediated neurodegeneration in Alzheimer's disease and related disorders. Nat Rev Neurosci 8:663-672.

Berry RW, Quinn B, Johnson N, Cochran EJ, Ghoshal N, Binder LI (2001) Pathological glial tau accumulations in neurodegenerative disease: review and case report. Neurochem Int 39:469-479.
Bonini NM, Fortini ME (2003) Human neurodegenerative disease modeling using Drosophila. Annu Rev Neurosci 26:627-656.

Booth GE, Kinrade EF, Hidalgo A (2000) Glia maintain follower neuron survival during Drosophila CNS development. Development 127:237-244.

Brand AH, Perrimon N (1993) Targeted gene expression as a means of altering cell fates and generating dominant phenotypes. Development 118:401-415.

Brandt R, Gergou A, Wacker I, Fath T, Hutter H (2009) A Caenorhabditis elegans model of tau hyperphosphorylation: induction of developmental defects by transgenic overexpression of Alzheimer's disease-like modified tau. Neurobiol Aging 30:22-33.

Brown S, Zeidler MP, Hombría JE (2006) JAK/STAT signalling in Drosophila controls cell motility during germ cell migration. Dev Dyn 235:958-966.

Buée L, Bussière T, Buée-Scherrer V, Delacourte A, Hof PR (2000) Tau protein isoforms, phosphorylation and role in neurodegenerative disorders. Brain Res Brain Res Rev 33:95-130.

Callus BA, Mathey-Prevot B (2002) SOCS36E, a novel Drosophila SOCS protein, suppresses JAK/STAT and EGF-R signalling in the imaginal wing disc. Oncogene 21:4812-4821.

Campbell G, Göring H, Lin T, Spana E, Andersson S, Doe CQ, Tomlinson A (1994) RK2, a glial-specific homeodomain protein required for embryonic nerve cord condensation and viability in Drosophila. Development 120:2957-2966.

Chen L, Feany MB (2005) Alpha-synuclein phosphorylation controls neurotoxicity and inclusion formation in a Drosophila model of Parkinson disease. Nat Neurosci 8:657-663.

Chin SS, Goldman JE (1996) Glial inclusions in CNS degenerative diseases. J Neuropathol Exp Neurol 55:499-508.

Conrad C, Andreadis A, Trojanowski JQ, Dickson DW, Kang D, Chen X, Wiederholt W, Hansen L, Masliah E, Thal LJ, Katzman R, Xia Y, Saitoh T (1997) Genetic evidence for the involvement of tau in progressive supranuclear palsy. Ann Neurol 41:277-281.

Dabir DV, Robinson MB, Swanson E, Zhang B, Trojanowski JQ, Lee VM, Forman MS (2006) Impaired glutamate transport in a mouse model of tau pathology in astrocytes. J Neurosci 26:644-654.

Dias-Santagata D, Fulga TA, Duttaroy A, Feany MB (2007) Oxidative stress mediates tau-induced neurodegeneration in Drosophila. J Clin Invest 117:236-245.

D’Souza I, Poorkaj P, Hong M, Nochlin D, Lee VM, Bird TD, Schellenberg GD (1999) Missense and silent tau gene mutations cause frontotemporal dementia with parkinsonism-chromosome 17 type, by affecting multiple alternative RNA splicing regulatory elements. Proc Natl Acad Sci U S A 96:5598-5603.

Feany MB, Dickson DW (1995) Widespread cytoskeletal pathology characterizes corticobasal degeneration. Am J Pathol 146:1388-1396.

Feany MB, Dickson DW (1996) Neurodegenerative disorders with extensive tau pathology: a comparative study and review. Ann Neurol 40:139-148.

Forman MS, Lal D, Zhang B, Dabir DV, Swanson E, Lee VM, Trojanowski JQ (2005) Transgenic mouse model of tau pathology in astrocytes leading to nervous system degeneration. J Neurosci 25:3539-3550.

Freeman MR, Doherty J (2006) Glial cell biology in Drosophila and vertebrates. Trends Neurosci 29:82-90.

Freeman MR, Delrow J, Kim J, Johnson E, Doe CQ (2003) Unwrapping glial biology: $\mathrm{Gcm}$ target genes regulating glial development, diversification, and function. Neuron 38:567-580.

Fulga TA, Elson-Schwab I, Khurana V, Steinhilb ML, Spires TL, Hyman BT, Feany MB (2007) Abnormal bundling and accumulation of F-actin mediates tau-induced neuronal degeneration in vivo. Nat Cell Biol 9:139-148.

Goedert M, Jakes R (2005) Mutations causing neurodegenerative tauopathies. Biochim Biophys Acta 1739:240-250.

Goedert M, Spillantini MG, Cairns NJ, Crowther RA (1992) Tau proteins of Alzheimer paired helical filaments: abnormal phosphorylation of all six brain isoforms. Neuron 8:159-168.

Heidary G, Fortini ME (2001) Identification and characterization of the Drosophila tau homolog. Mech Dev 108:171-178.

Heneka MT, O’Banion MK (2007) Inflammatory processes in Alzheimer's disease. J Neuroimmunol 184:69-91.

Higuchi M, Zhang B, Forman MS, Yoshiyama Y, Trojanowski JQ, Lee VM (2005) Axonal degeneration induced by targeted expression of mutant human tau in oligodendrocytes of transgenic mice that model glial tauopathies. J Neurosci 25:9434-9443.

Houlden H, Baker M, Morris HR, MacDonald N, Pickering-Brown S, Adam- 
son J, Lees AJ, Rossor MN, Quinn NP, Kertesz A, Khan MN, Hardy J, Lantos PL, St George-Hyslop P, Munoz DG, Mann D, Lang AE, Bergeron C, Bigio EH, Litvan I, Bhatia KP, Dickson D, Wood NW, Hutton M (2001) Corticobasal degeneration and progressive supranuclear palsy share a common tau haplotype. Neurology 56:1702-1706.

Hutton M, Lendon CL, Rizzu P, Baker M, Froelich S, Houlden H, PickeringBrown S, Chakraverty S, Isaacs A, Grover A, Hackett J, Adamson J, Lincoln S, Dickson D, Davies P, Petersen RC, Stevens M, de Graaff E, Wauters E, van Baren J, et al. (1998) Association of missense and 5'splice-site mutations in tau with the inherited dementia FTDP-17. Nature 393:702-705.

Ikeda K, Akiyama H, Kondo H, Haga C, Tanno E, Tokuda T, Ikeda S (1995) Thorn-shaped astrocytes: possibly secondarily induced tau-positive glial fibrillary tangles. Acta Neuropathol 90:620-625.

Ishihara T, Hong M, Zhang B, Nakagawa Y, Lee MK, Trojanowski JQ, Lee VM (1999) Age-dependent emergence and progression of a tauopathy in transgenic mice overexpressing the shortest human tau isoform. Neuron 24:751-762.

Ito K, Arai K, Yoshiyama Y, Kashiwado K, Sakakibara Y, Hattori T (2008) Astrocytic tau pathology positively correlates with neurofibrillary tangle density in progressive supranuclear palsy. Acta Neuropathol 115:623-628.

Jackson GR, Wiedau-Pazos M, Sang TK, Wagle N, Brown CA, Massachi S, Geschwind DH (2002) Human wild-type tau interacts with wingless pathway components and produces neurofibrillary pathology in Drosophila. Neuron 34:509-519.

Khurana V, Lu Y, Steinhilb ML, Oldham S, Shulman JM, Feany MB (2006) TOR-mediated cell-cycle activation causes neurodegeneration in a Drosophila tauopathy model. Curr Biol 16:230-241.

Kosik KS, Joachim CL, Selkoe DJ (1986) Microtubule-associated protein tau (tau) is a major antigenic component of paired helical filaments in Alzheimer disease. Proc Natl Acad Sci U S A 83:4044-4048.

Kraemer BC, Zhang B, Leverenz JB, Thomas JH, Trojanowski JQ, Schellenberg GD (2003) Neurodegeneration and defective neurotransmission in a Caenorhabditis elegans model of tauopathy. Proc Natl Acad Sci U S A 100:9980-9985.

Ksiezak-Reding H, Morgan K, Mattiace LA, Davies P, Liu WK, Yen SH, Weidenheim K, Dickson DW (1994) Ultrastructure and biochemical composition of paired helical filaments in corticobasal degeneration. Am J Pathol 145:1496-1508.

Lai SL, Lee T (2006) Genetic mosaic with dual binary transcriptional systems in Drosophila. Nat Neurosci 9:703-709.

Lewis J, McGowan E, Rockwood J, Melrose H, Nacharaju P, Van Slegtenhorst M, Gwinn-Hardy K, Paul Murphy M, Baker M, Yu X, Duff K, Hardy J, Corral A, Lin WL, Yen SH, Dickson DW, Davies P, Hutton M (2000) Neurofibrillary tangles, amyotrophy and progressive motor disturbance in mice expressing mutant (P301L) tau protein. Nat Genet 25:402-405.

Liévens JC, Rival T, Iché M, Chneiweiss H, Birman S (2005) Expanded polyglutamine peptides disrupt EGF receptor signaling and glutamate transporter expression in Drosophila. Hum Mol Genet 14:713-724.

MacDonald JM, Beach MG, Porpiglia E, Sheehan AE, Watts RJ, Freeman MR (2006) The Drosophila cell corpse engulfment receptor Draper mediates glial clearance of severed axons. Neuron 50:869-881.

Marsh JL, Thompson LM (2006) Drosophila in the study of neurodegenerative disease. Neuron 52:169-178.

Martínez A, Carmona M, Portero-Otin M, Naudí A, Pamplona R, Ferrer I (2008) Type-dependent oxidative damage in frontotemporal lobar degeneration: cortical astrocytes are targets of oxidative damage. J Neuropathol Exp Neurol 67:1122-1136.

McGuire SE, Le PT, Osborn AJ, Matsumoto K, Davis RL (2003) Spatiotemporal rescue of memory dysfunction in Drosophila. Science 302:1765-1768.

Mercken M, Fischer I, Kosik KS, Nixon RA (1995) Three distinct axonal transport rates for tau, tubulin, and other microtubule-associated proteins: evidence for dynamic interactions of tau with microtubules in vivo. J Neurosci 15:8259-8267.

Mirra SS, Murrell JR, Gearing M, Spillantini MG, Goedert M, Crowther RA, Levey AI, Jones R, Green J, Shoffner JM, Wainer BH, Schmidt ML, Trojanowski JQ, Ghetti B (1999) Tau pathology in a family with dementia and a P301L mutation in tau. J Neuropathol Exp Neurol 58:335-345.

Miyasaka T, Ding Z, Gengyo-Ando K, Oue M, Yamaguchi H, Mitani S, Ihara Y (2005) Progressive neurodegeneration in C. elegans model of tauopathy. Neurobiol Dis 20:372-383.
Morales-Corraliza J, Mazzella MJ, Berger JD, Diaz NS, Choi JH, Levy E, Matsuoka Y, Planel E, Mathews PM (2009) In vivo turnover of tau and APP metabolites in the brains of wild-type and Tg2576 mice: greater stability of sAPP in the beta-amyloid depositing mice. PLoS One 4:e7134.

Muntané G, Dalfó E, Martínez A, Rey MJ, Avila J, Pérez M, Portero M, Pamplona R, Ayala V, Ferrer I (2006) Glial fibrillary acidic protein is a major target of glycoxidative and lipoxidative damage in Pick's disease. J Neurochem 99:177-185.

Muqit MM, Feany MB (2002) Modelling neurodegenerative diseases in Drosophila: a fruitful approach? Nat Rev Neurosci 3:237-243.

Nakano I, Iwatsubo T, Otsuka N, Kamei M, Matsumura K, Mannen T (1992) Paired helical filaments in astrocytes: electron microscopy and immunohistochemistry in a case of atypical Alzheimer's disease. Acta Neuropathol 83:228-232.

Nishimura I, Yang Y, Lu B (2004) PAR-1 kinase plays an initiator role in a temporally ordered phosphorylation process that confers tau toxicity in Drosophila. Cell 116:671-682.

Nishimura M, Tomimoto H, Suenaga T, Namba Y, Ikeda K, Akiguchi I, Kimura J (1995) Immunocytochemical characterization of glial fibrillary tangles in Alzheimer's disease brain. Am J Pathol 146:1052-1058.

Ozog MA, Bernier SM, Bates DC, Chatterjee B, Lo CW, Naus CC (2004) The complex of ciliary neurotrophic factor-ciliary neurotrophic factor receptor alpha up-regulates connexin 43 and intercellular coupling in astrocytes via the Janus tyrosine kinase/signal transducer and activator of transcription pathway. Mol Biol Cell 15:4761-4774.

Papasozomenos SC (1989a) Tau protein immunoreactivity in dementia of the Alzheimer type. I. Morphology, evolution, distribution, and pathogenetic implications. Lab Invest 60:123-137.

Papasozomenos SC (1989b) Tau protein immunoreactivity in dementia of the Alzheimer type. II. Electron microscopy and pathogenetic implications. Effects of fixation on the morphology of the Alzheimer's abnormal filaments. Lab Invest 60:375-389.

Pascual A, Hidalgo-Figueroa M, Piruat JI, Pintado CO, Gómez-Díaz R, López-Barneo J (2008) Absolute requirement of GDNF for adult catecholaminergic neuron survival. Nat Neurosci 11:755-761.

Periquet M, Fulga T, Myllykangas L, Schlossmacher MG, Feany MB (2007) Aggregated $\alpha$-synuclein mediates dopaminergic neurotoxicity in vivo. J Neurosci 27:3338-3346.

Perrimon N, Mahowald AP (1986) 1(1)hopscotch, A larval-pupal zygotic lethal with a specific maternal effect on segmentation in Drosophila. Dev Biol 118:28-41.

Probst A, Götz J, Wiederhold KH, Tolnay M, Mistl C, Jaton AL, Hong M, Ishihara T, Lee VM, Trojanowski JQ, Jakes R, Crowther RA, Spillantini MG, Bürki K, Goedert M (2000) Axonopathy and amyotrophy in mice transgenic for human four-repeat tau protein. Acta Neuropathol 99:469-481.

Ransom B, Behar T, Nedergaard M (2003) New roles for astrocytes (stars at last). Trends Neurosci 26:520-522.

Rival T, Soustelle L, Strambi C, Besson MT, Iché M, Birman S (2004) Decreasing glutamate buffering capacity triggers oxidative stress and neuropil degeneration in the Drosophila brain. Curr Biol 14:599-605.

Santacruz K, Lewis J, Spires T, Paulson J, Kotilinek L, Ingelsson M, Guimaraes A, DeTure M, Ramsden M, McGowan E, Forster C, Yue M, Orne J, Janus C, Mariash A, Kuskowski M, Hyman B, Hutton M, Ashe KH (2005) Tau suppression in a neurodegenerative mouse model improves memory function. Science 309:476-481.

Schultz C, Ghebremedhin E, Del Tredici K, Rüb U, Braak H (2004) High prevalence of thorn-shaped astrocytes in the aged human medial temporal lobe. Neurobiol Aging 25:397-405.

Schwartz JP, Nishiyama N (1994) Neurotrophic factor gene expression in astrocytes during development and following injury. Brain Res Bull 35:403-407.

Sepp KJ, Auld VJ (1999) Conversion of lacZ enhancer trap lines to GAL4 lines using targeted transposition in Drosophila melanogaster. Genetics 151:1093-1101.

Sepp KJ, Schulte J, Auld VJ (2001) Peripheral glia direct axon guidance across the CNS/PNS transition zone. Dev Biol 238:47-63.

Spillantini MG, Bird TD, Ghetti B (1998) Frontotemporal dementia and Parkinsonism linked to chromosome 17: a new group of tauopathies. Brain Pathol 8:387-402.

Spires TL, Orne JD, SantaCruz K, Pitstick R, Carlson GA, Ashe KH, Hyman BT 
(2006) Region-specific dissociation of neuronal loss and neurofibrillary pathology in a mouse model of tauopathy. Am J Pathol 168:1598-1607.

Spittaels K, Van den Haute C, Van Dorpe J, Bruynseels K, Vandezande K, Laenen I, Geerts H, Mercken M, Sciot R, Van Lommel A, Loos R, Van Leuven F (1999) Prominent axonopathy in the brain and spinal cord of transgenic mice overexpressing four-repeat human tau protein. Am J Pathol 155:2153-2165.

Sriram K, Benkovic SA, Hebert MA, Miller DB, O'Callaghan JP (2004) Induction of gp130-related cytokines and activation of JAK2/STAT3 pathway in astrocytes precedes up-regulation of glial fibrillary acidic protein in the 1-methyl-4-phenyl-1,2,3,6-tetrahydropyridine model of neurodegeneration: key signaling pathway for astrogliosis in vivo? J Biol Chem 279:19936-19947.

Steffan JS, Bodai L, Pallos J, Poelman M, McCampbell A, Apostol BL, Kazantsev A, Schmidt E, Zhu YZ, Greenwald M, Kurokawa R, Housman DE, Jackson GR, Marsh JL, Thompson LM (2001) Histone deacetylase inhibitors arrest polyglutamine-dependent neurodegeneration in Drosophila. Nature 413:739-743.

Steinhilb ML, Dias-Santagata D, Fulga TA, Felch DL, Feany MB (2007a) Tau phosphorylation sites work in concert to promote neurotoxicity in vivo. Mol Biol Cell 18:5060-5068.

Steinhilb ML, Dias-Santagata D, Mulkearns EE, Shulman JM, Biernat J, Mandelkow EM, Feany MB (2007b) S/P and T/P phosphorylation is critical for tau neurotoxicity in Drosophila. J Neurosci Res 85:1271-1278.

Sun B, Wang W, Salvaterra PM (1998) Functional analysis and tissuespecific expression of Drosophila $\mathrm{Na}^{+}-\mathrm{K}^{+}$-ATPase subunits. J Neurochem 71:142-151.

Tix S, Eule E, Fischbach KF, Benzer S (1997) Glia in the chiasms and medulla of the Drosophila melanogaster optic lobes. Cell Tissue Res 289: 397-409.

Togo T, Dickson DW (2002) Tau accumulation in astrocytes in progressive supranuclear palsy is a degenerative rather than a reactive process. Acta Neuropathol 104:398-402.

Tolnay M, Grazia Spillantini M, Rizzini C, Eccles D, Lowe J, Ellison D (2000)
A new case of frontotemporal dementia and parkinsonism resulting from an intron $10+3$-splice site mutation in the tau gene: clinical and pathological features. Neuropathol Appl Neurobiol 26:368-378.

Warrick JM, Chan HY, Gray-Board GL, Chai Y, Paulson HL, Bonini NM (1999) Suppression of polyglutamine-mediated neurodegeneration in Drosophila by the molecular chaperone HSP70. Nat Genet 23:425-428.

Wilczynska KM, Singh SK, Adams B, Bryan L, Rao RR, Valerie K, Wright S, Griswold-Prenner I, Kordula T (2009) Nuclear factor I isoforms regulate gene expression during the differentiation of human neural progenitors to astrocytes. Stem Cells 27:1173-1181.

Wittmann CW, Wszolek MF, Shulman JM, Salvaterra PM, Lewis J, Hutton M, Feany MB (2001) Tauopathy in Drosophila: neurodegeneration without neurofibrillary tangles. Science 293:711-714.

Xiong WC, Montell C (1995) Defective glia induce neuronal apoptosis in the repo visual system of Drosophila. Neuron 14:581-590.

Xiong WC, Okano H, Patel NH, Blendy JA, Montell C (1994) repo encodes a glial-specific homeo domain protein required in the Drosophila nervous system. Genes Dev 8:981-994.

Yamamoto T, Hirano A (1986) A comparative study of modified Bielschowsky, Bodian and thioflavin S stains on Alzheimer's neurofibrillary tangles. Neuropathol Appl Neurobiol 12:3-9.

Yamanaka T, Miyazaki H, Oyama F, Kurosawa M, Washizu C, Doi H, Nukina N (2008) Mutant Huntingtin reduces HSP70 expression through the sequestration of NF-Y transcription factor. EMBO J 27:827-839.

Yasuyama K, Kitamoto T, Salvaterra PM (1995) Immunocytochemical study of choline acetyltransferase in Drosophila melanogaster: an analysis of cis-regulatory regions controlling expression in the brain of cDNAtransformed flies. J Comp Neurol 361:25-37.

Yoshimura A, Naka T, Kubo M (2007) SOCS proteins, cytokine signalling and immune regulation. Nat Rev Immunol 7:454-465.

Ziegenfuss JS, Biswas R, Avery MA, Hong K, Sheehan AE, Yeung YG, Stanley ER, Freeman MR (2008) Draper-dependent glial phagocytic activity is mediated by Src and Syk family kinase signalling. Nature 453:935939. 